CRYSTALLOGRAPHIC COMMUNICATIONS

ISSN 2056-9890

Received 7 May 2017

Accepted 19 May 2017

Edited by M. Weil, Vienna University of

Technology, Austria

Keywords: crystal structure; $\mathrm{CaNi}_{2} \mathrm{Fe}\left(\mathrm{PO}_{4}\right)_{3}$; transition metal phosphate; solid-state reactions; $\alpha-\mathrm{CrPO}_{4}$ structure type.

CCDC reference: 1551182

Supporting information: this article has supporting information at journals.iucr.org/e

\section{Crystal structure of calcium dinickel(II) iron(III) tris(orthophosphate): $\mathrm{CaNi}_{2} \mathrm{Fe}\left(\mathrm{PO}_{4}\right)_{3}$}

\author{
Said Ouaatta,* Abderrazzak Assani, Mohamed Saadi and Lahcen El Ammari \\ Laboratoire de Chimie du Solide Appliquée, Faculty of Sciences, Mohammed V University in Rabat, Avenue Ibn Battouta, \\ BP 1014, Rabat, Morocco. *Correspondence e-mail: saidouaatta87@gmail.com
}

The title compound, $\mathrm{CaNi}_{2} \mathrm{Fe}\left(\mathrm{PO}_{4}\right)_{3}$, was synthesized by solid-state reactions. Its structure is closely related to that of $\alpha-\mathrm{CrPO}_{4}$ in the space group Imma. Except for two $\mathrm{O}$ atoms in general positions, all atoms are located in special positions. The three-dimensional framework is built up from two types of sheets extending parallel to (100). The first sheet is made up from two edge-sharing $\left[\mathrm{NiO}_{6}\right]$ octahedra, leading to the formation of $\left[\mathrm{Ni}_{2} \mathrm{O}_{10}\right]$ double octahedra that are connected to two $\mathrm{PO}_{4}$ tetrahedra through a common edge and corners. The second sheet results from rows of corner-sharing $\left[\mathrm{FeO}_{6}\right]$ octahedra and $\mathrm{PO}_{4}$ tetrahedra forming an infinite linear chain. These layers are linked together through common corners of $\mathrm{PO}_{4}$ tetrahedra and $\left[\mathrm{FeO}_{6}\right]$ octahedra, resulting in an open three-dimensional framework that delimits two types of channels parallel to [100] and [010] in which the eightfold-coordinated $\mathrm{Ca}^{\mathrm{II}}$ cations are located.

\section{Chemical context}

Phosphates belonging to the alluaudite (Moore, 1971) or to the $\alpha-\mathrm{CrPO}_{4}$ (Attfield et al., 1988) structure type exhibit interesting physical and chemical properties. Consequently, these compounds have many promising applications such as use as positive electrodes in lithium and sodium batteries (Kim et al., 2014; Huang et al., 2015) or as catalysts (Kacimi et al., 2005). Over the last few years, phosphate-based compounds crystallizing in the $\alpha-\mathrm{CrPO}_{4}$ or alluaudite structure types have been investigated by us. In this context, new phosphates adopting the alluaudite or $\alpha-\mathrm{CrPO}_{4}$ structure type have been synthesized and structurally characterized. For example, the mixed-valence manganese phosphates $\mathrm{PbMn}_{2}^{\mathrm{II}} \mathrm{Mn}^{\mathrm{III}}\left(\mathrm{PO}_{4}\right)_{3}$ (Alhakmi et al., 2013) and $\mathrm{PbMn}_{2}^{\mathrm{II}}{ }_{2} \mathrm{Mn}^{\mathrm{III}}\left(\mathrm{PO}_{4}\right)_{3}$ (Assani et al., 2013), the magnesium phosphate $\mathrm{NaMg}_{3}\left(\mathrm{PO}_{4}\right)\left(\mathrm{HPO}_{4}\right)_{2}($ Ould Saleck et al., 2015) and silver nickel phosphate $\mathrm{Ag}_{2} \mathrm{Ni}_{3}\left(\mathrm{HPO}_{4}\right)$ $\left(\mathrm{PO}_{4}\right)_{2}$ (Assani et al., 2011) were synthesized by hydrothermal methods, while solid-state reactions were applied to synthesize $\mathrm{SrNi}_{2} \mathrm{Fe}\left(\mathrm{PO}_{4}\right)_{3}$ (Ouaatta et al., 2015) and $\mathrm{Na}_{2} \mathrm{Co}_{2} \mathrm{Fe}\left(\mathrm{PO}_{4}\right)_{3}$ (Bouraima et al., 2015). In a continuation of the latter preparation route, we have investigated pseudo-quaternary systems $M \mathrm{O}-\mathrm{NiO}-\mathrm{Fe}_{2} \mathrm{O}_{3}-\mathrm{P}_{2} \mathrm{O}_{5}$ ( $M$ represents a divalent cation) and report here on the synthesis and crystal structure of the title compound, $\mathrm{CaNi}_{2} \mathrm{Fe}\left(\mathrm{PO}_{4}\right)_{3}$.

\section{Structural commentary}

$\mathrm{CaNi}_{2} \mathrm{Fe}\left(\mathrm{PO}_{4}\right)_{3}$ crystallizes in the $\alpha-\mathrm{CrPO}_{4}$ structure type. The principal building units of the crystal structure are one $\left[\mathrm{CaO}_{8}\right]$ polyhedron, $\left[\mathrm{FeO}_{6}\right]$ and $\left[\mathrm{NiO}_{6}\right]$ octahedra and $\mathrm{PO}_{4}$ tetrahedra, 


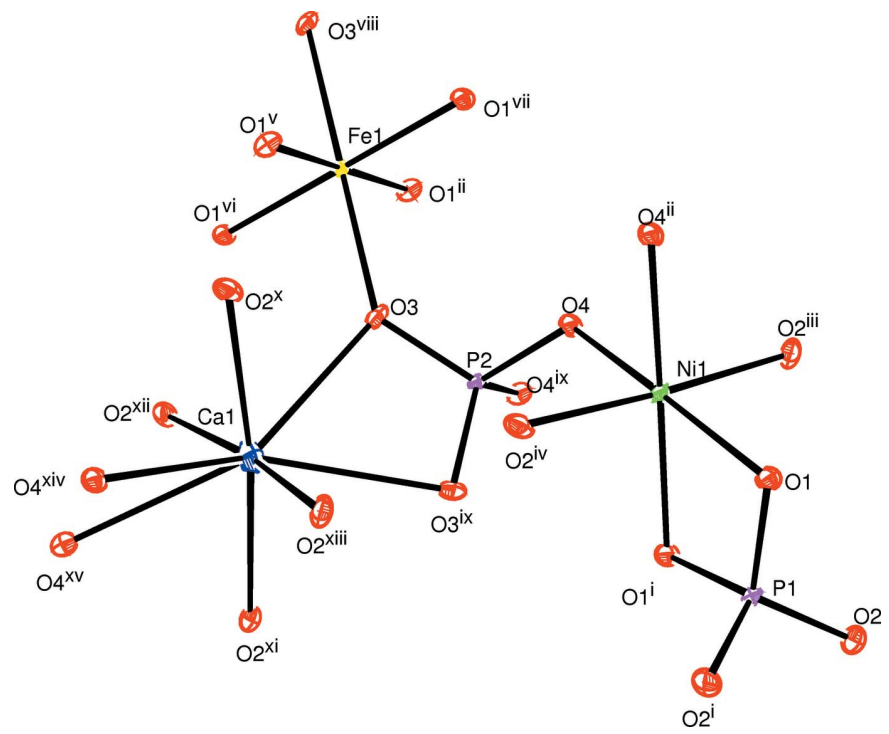

Figure 1

The principal building units in the crystal structure of the title compound. Displacement ellipsoids are drawn at the $50 \%$ probability level. [Symmetry codes: (i) $-x+2,-y+\frac{3}{2}, z+1$; (ii) $x, y, z+1$; (iii) $-x+2$, $-y+\frac{3}{2}, z$; (iv) $-x+\frac{3}{2},-y+1, z+\frac{1}{2}$; (v) $x+\frac{1}{2}, y+\frac{1}{2}, z+\frac{1}{2}$; (vi) $-x+\frac{3}{2}, y+\frac{1}{2}$, $z+\frac{1}{2}$; (vii) $x+\frac{1}{2},-y+1, z+\frac{1}{2}$; (viii) $-x+\frac{3}{2},-y+\frac{3}{2},-z+\frac{1}{2}$; (ix) $-x+\frac{3}{2}, y$, $-z+\frac{1}{2} ;(x) x,-y+1,-z$; (xi) $-x+1, y, z$; (xii) $x,-y+1,-z+1$; (xiii) $-x+1,-y+1,-z+1$; (xiv) $x-\frac{1}{2}, y,-z+\frac{1}{2}$.]

as shown in Fig. 1.The octahedral coordination sphere of the iron(III) cation is more distorted than that of nickel(II), with $\mathrm{Fe}-\mathrm{O}$ bond lengths in the range 1.9504 (7)-2.0822 (11) $\AA$ and $\mathrm{Ni}-\mathrm{O}$ bond lengths in the range 2.0498 (8)-2.0841 (8) $\AA$. In the title structure, all atoms are on special positions, except for the two oxygen atoms $\mathrm{O} 1$ and $\mathrm{O} 2$, which are on general positions. The structure can be described by the stacking of two types of sheets extending parallel to (100). The first sheet is formed by alternating $\left[\mathrm{FeO}_{6}\right]$ octahedra and $\mathrm{PO}_{4}$ tetrahedra sharing corners to build a linear infinite chain surrounding a zigzag chain of $\mathrm{Ca}^{\mathrm{II}+}$ cations (Fig. 2). The second sheet is built up from two edge-sharing $\left[\mathrm{NiO}_{6}\right]$ octahedra leading to the formation of $\left[\mathrm{Ni}_{2} \mathrm{O}_{10}\right]$ double octahedra, which are connected to two $\mathrm{PO}_{4}$ tetrahedra by a common edge and a common corner, as shown in Fig. 3. The linkage of both layers, through vertices of $\mathrm{PO}_{4}$ tetrahedra and $\left[\mathrm{FeO}_{6}\right]$ octahedra, gives rise to the formation of an open three-dimensional framework that delimits two types of channels parallel to [100] and [010] in which the $\mathrm{Ca}^{\mathrm{II}}$ cations are located with eight neighbouring $\mathrm{O}$

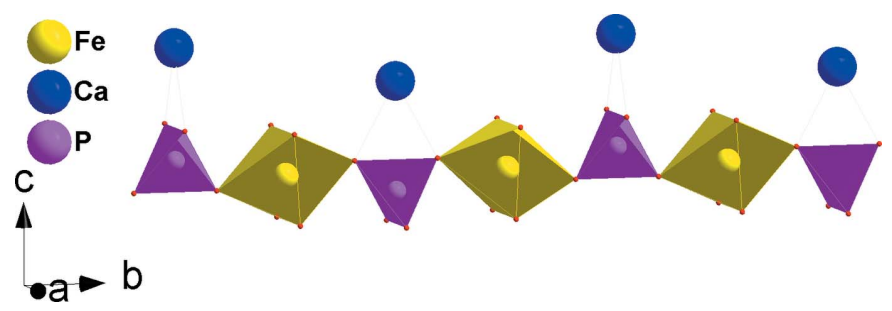

Figure 2

A chain formed by sharing corners of $\mathrm{PO}_{4}$ tetrahedra and $\left[\mathrm{FeO}_{6}\right]$ octahedra, alternating with a zigzag chain of calcium cations.

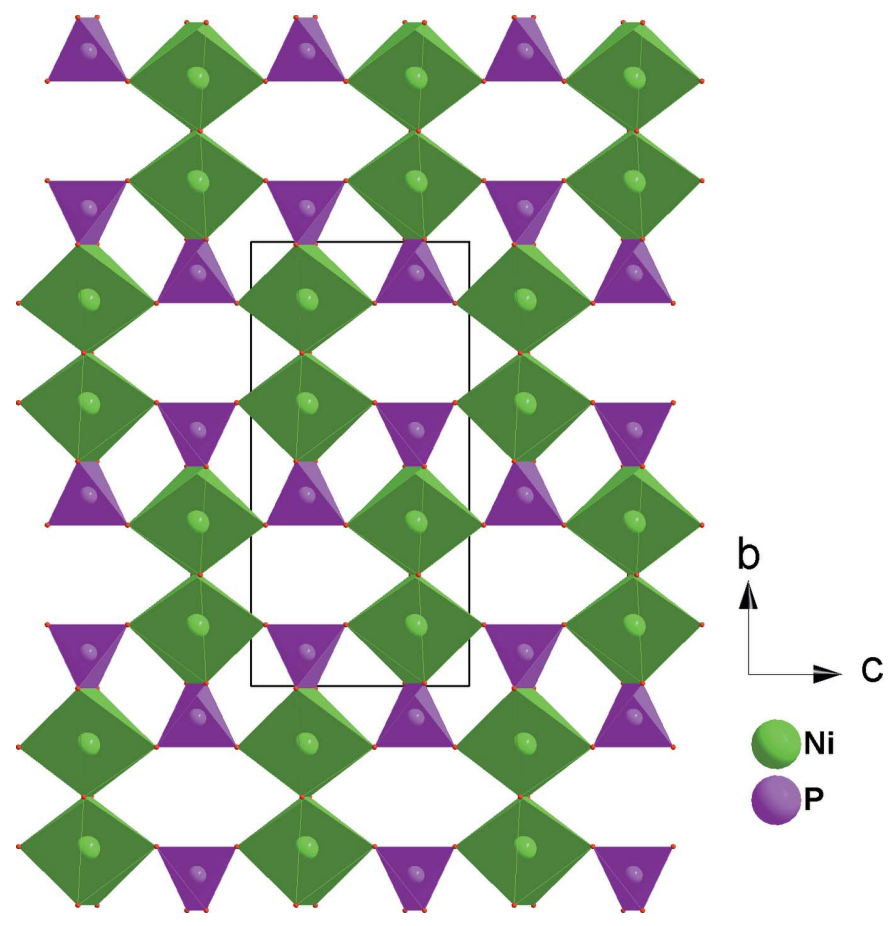

Figure 3

Edge-sharing $\left[\mathrm{NiO}_{6}\right]$ octahedra linked by $\mathrm{PO}_{4}$ tetrahedra, forming a sheet parallel to $(100)$.

atoms, as shown in Fig. 4. The title compound has a stoichiometric composition like that of the related strontium homologue $\mathrm{SrNi}_{2} \mathrm{Fe}\left(\mathrm{PO}_{4}\right)_{3}$.

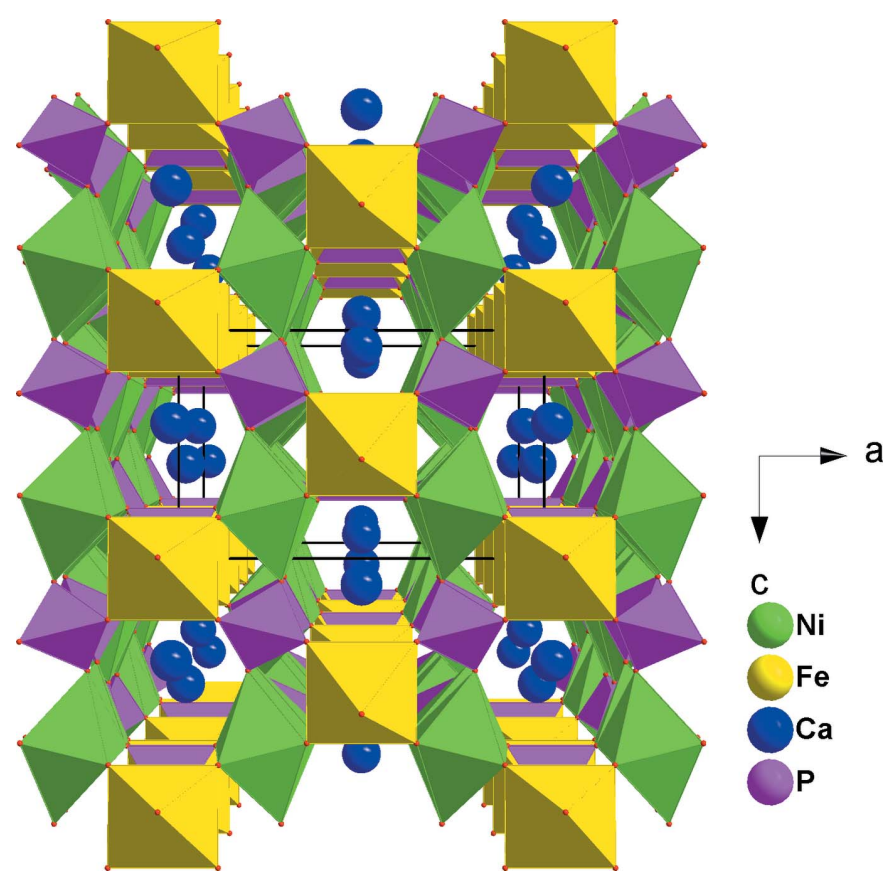

Figure 4

Polyhedral representation of $\mathrm{CaNiO}_{2} \mathrm{Fe}\left(\mathrm{PO}_{4}\right)_{3}$, showing channels running parallel to [100]. 


\section{Synthesis and crystallization}

$\mathrm{CaNi}_{2} \mathrm{Fe}\left(\mathrm{PO}_{4}\right)_{3}$ was prepared by solid-state reactions in air. Stoichiometric mixtures of calcium, nickel and iron precursors were dissolved in water to which $85 \%_{\text {wt }}$ phosphoric acid was added. The obtained mixture was stirred without heating for $24 \mathrm{~h}$ and was subsequently evaporated to dryness at $343 \mathrm{~K}$. The resulting dry residue was ground in an agate mortar until homogeneity, progressively heated in a platinum crucible up to $873 \mathrm{~K}$ to remove the volatile decomposition products, and then melted at $1433 \mathrm{~K}$. The molten product was cooled down slowly with a $5 \mathrm{~K} \mathrm{~h}^{-1}$ rate and then to room temperature. The crystals obtained after washing with water were orange with parallelepipedal forms.

\section{Refinement}

Crystal data, data collection and structure refinement details are summarized in Table 1 . The maximum and minimum remaining electron densities are 0.68 and $0.41 \AA$, respectively, away from the Ni1 site.

\section{Acknowledgements}

The authors thank the Unit of Support for Technical and Scientific Research (UATRS, CNRST) for the X-ray measurements.

\section{Funding information}

Funding for this research was provided by: Mohammed V University, Rabat, Morocco.

\section{References}

Alhakmi, G., Assani, A., Saadi, M. \& El Ammari, L. (2013). Acta Cryst. E69, i40.

Assani, A., El Ammari, L., Zriouil, M. \& Saadi, M. (2011). Acta Cryst. E67, i40.

Assani, A., Saadi, M., Alhakmi, G., Houmadi, E. \& El Ammari, L. (2013). Acta Cryst. E69, i60.

Attfield, J. P., Cheetham, A. K., Cox, D. E. \& Sleight, A. W. (1988). J. Appl. Cryst. 21, 452-457.

Bouraima, A., Assani, A., Saadi, M., Makani, T. \& El Ammari, L. (2015). Acta Cryst. E71, 558-560.

Brandenburg, K. (2006). DIAMOND. Crystal Impact GbR, Bonn, Germany.
Table 1

Experimental details.

\begin{tabular}{|c|c|}
\hline \multicolumn{2}{|l|}{ Crystal data } \\
\hline Chemical formula & $\mathrm{CaNi}_{2} \mathrm{Fe}\left(\mathrm{PO}_{4}\right)_{3}$ \\
\hline$M_{\mathrm{r}}$ & 498.26 \\
\hline Crystal system, space group & Orthorhombic, Imma \\
\hline Temperature (K) & 296 \\
\hline$a, b, c(\AA)$ & $10.3126(3), 13.1138(3), 6.4405(2)$ \\
\hline$V\left(\AA^{3}\right)$ & $871.00(4)$ \\
\hline$Z$ & 4 \\
\hline Radiation type & Мo $K \alpha$ \\
\hline$\mu\left(\mathrm{mm}^{-1}\right)$ & 7.14 \\
\hline Crystal size $(\mathrm{mm})$ & $0.30 \times 0.27 \times 0.21$ \\
\hline \multicolumn{2}{|l|}{ Data collection } \\
\hline Diffractometer & Bruker X8 APEX \\
\hline Absorption correction & $\begin{array}{l}\text { Multi-scan (SADABS; Krause et } \\
\quad \text { al., 2015) }\end{array}$ \\
\hline$T_{\min }, T_{\max }$ & $0.596,0.748$ \\
\hline $\begin{array}{l}\text { No. of measured, independent and } \\
\text { observed }[I>2 \sigma(I)] \text { reflections }\end{array}$ & $8446,1171,1153$ \\
\hline$R_{\text {int }}$ & 0.020 \\
\hline$(\sin \theta / \lambda)_{\max }\left(\AA^{-1}\right)$ & 0.840 \\
\hline \multicolumn{2}{|l|}{ Refinement } \\
\hline$R\left[F^{2}>2 \sigma\left(F^{2}\right)\right], w R\left(F^{2}\right), S$ & $0.017,0.044,1.17$ \\
\hline No. of reflections & 1171 \\
\hline No. of parameters & 54 \\
\hline$\Delta \rho_{\max }, \Delta \rho_{\min }\left(\mathrm{e} \AA^{-3}\right)$ & $0.76,-0.63$ \\
\hline
\end{tabular}

Computer programs: APEX2 and SAINT (Bruker, 2009), SHELXT2014 (Sheldrick, 2015a), SHELXL2014 (Sheldrick, 2015b), ORTEP-3 for Windows (Farrugia, 2012), DIAMOND (Brandenburg, 2006) and publCIF (Westrip, 2010).

Bruker (2009). APEX2 and SAINT. Bruker AXS Inc., Madison, Wisconsin, USA.

Farrugia, L. J. (2012). J. Appl. Cryst. 45, 849-854.

Huang, W., Li, B., Saleem, M. F., Wu, X., Li, J., Lin, J., Xia, D., Chu, W. \& Wu, Z. (2015). Chem. Eur. J. 21, 851-860.

Kacimi, M., Ziyad, M. \& Hatert, F. (2005). Mater. Res. Bull. 40, 682693.

Kim, J., Kim, H., Park, K.-Y., Park, Y.-U., Lee, S., Kwon, H.-S., Yoo, H.-I. \& Kang, K. (2014). J. Mater. Chem. A, 2, 8632-8636.

Krause, L., Herbst-Irmer, R., Sheldrick, G. M. \& Stalke, D. (2015). J. Appl. Cryst. 48, 3-10.

Moore, P. B. (1971). Am. Mineral. 56, 1955-1975.

Ouaatta, S., Assani, A., Saadi, M. \& El Ammari, L. (2015). Acta Cryst. E71, 1255-1258.

Ould Saleck, A., Assani, A., Saadi, M., Mercier, C., Follet, C. \& El Ammari, L. (2015). Acta Cryst. E71, 813-815.

Sheldrick, G. M. (2015a). Acta Cryst. A71, 3-8.

Sheldrick, G. M. (2015b). Acta Cryst. C71, 3-8.

Westrip, S. P. (2010). J. Appl. Cryst. 43, 920-925. 


\section{supporting information}

Acta Cryst. (2017). E73, 893-895 [https://doi.org/10.1107/S2056989017007411]

Crystal structure of calcium dinickel(II) iron(III) tris(orthophosphate):

$\mathrm{CaNi} 2 \mathrm{Fe}\left(\mathrm{PO}_{4}\right)_{3}$

\section{Said Ouaatta, Abderrazzak Assani, Mohamed Saadi and Lahcen El Ammari}

Computing details

Data collection: APEX2 (Bruker, 2009); cell refinement: SAINT (Bruker, 2009); data reduction: SAINT (Bruker, 2009); program(s) used to solve structure: SHELXT2014 (Sheldrick, 2015a); program(s) used to refine structure: SHELXL2014 (Sheldrick, 2015b); molecular graphics: ORTEP-3 for Windows (Farrugia, 2012) and DIAMOND (Brandenburg, 2006); software used to prepare material for publication: publCIF (Westrip, 2010).

Calcium dinickel(II) iron(III) tris(orthophosphate)

\section{Crystal data}

$\mathrm{CaNi}_{2} \mathrm{Fe}\left(\mathrm{PO}_{4}\right)_{3}$

$M_{r}=498.26$

Orthorhombic, Imma

$a=10.3126(3) \AA$

$b=13.1138(3) \AA$

$c=6.4405(2) \AA$

$V=871.00(4) \AA^{3}$

$Z=4$

$F(000)=972$

\section{Data collection}

Bruker X8 APEX

diffractometer

Radiation source: fine-focus sealed tube

Graphite monochromator

$\varphi$ and $\omega$ scans

Absorption correction: multi-scan

(SADABS; Krause et al., 2015)

$T_{\min }=0.596, T_{\max }=0.748$

Refinement

Refinement on $F^{2}$

Least-squares matrix: full

$R\left[F^{2}>2 \sigma\left(F^{2}\right)\right]=0.017$

$w R\left(F^{2}\right)=0.044$

$S=1.17$

1171 reflections

54 parameters

0 restraints
$D_{\mathrm{x}}=3.800 \mathrm{Mg} \mathrm{m}^{-3}$

Mo $K \alpha$ radiation, $\lambda=0.71073 \AA$

Cell parameters from 1171 reflections

$\theta=3.1-36.6^{\circ}$

$\mu=7.14 \mathrm{~mm}^{-1}$

$T=296 \mathrm{~K}$

Parallelepiped, orange

$0.30 \times 0.27 \times 0.21 \mathrm{~mm}$

8446 measured reflections

1171 independent reflections

1153 reflections with $I>2 \sigma(I)$

$R_{\text {int }}=0.020$

$\theta_{\max }=36.6^{\circ}, \theta_{\min }=3.1^{\circ}$

$h=-16 \rightarrow 17$

$k=-20 \rightarrow 22$

$l=-10 \rightarrow 10$

$$
\begin{aligned}
& w=1 /\left[\sigma^{2}\left(F_{\mathrm{o}}^{2}\right)+(0.0216 P)^{2}+1.467 P\right] \\
& \quad \text { where } P=\left(F_{\mathrm{o}}^{2}+2 F_{\mathrm{c}}^{2}\right) / 3 \\
& (\Delta / \sigma)_{\max }=0.001 \\
& \Delta \rho_{\max }=0.76 \mathrm{e} \AA^{-3} \\
& \Delta \rho_{\min }=-0.63 \mathrm{e} \AA^{-3} \\
& \text { Extinction correction: SHELXL2014 } \\
& \quad(\text { Sheldrick, } 2015 b), \\
& \mathrm{Fc}^{*}=\mathrm{kFc}\left[1+0.001 \times \mathrm{xc}^{2} \lambda^{3} / \sin (2 \theta)\right]^{-1 / 4}
\end{aligned}
$$

Extinction coefficient: 0.0033 (2) 


\section{Special details}

Geometry. All esds (except the esd in the dihedral angle between two 1.s. planes) are estimated using the full covariance matrix. The cell esds are taken into account individually in the estimation of esds in distances, angles and torsion angles; correlations between esds in cell parameters are only used when they are defined by crystal symmetry. An approximate (isotropic) treatment of cell esds is used for estimating esds involving l.s. planes.

Fractional atomic coordinates and isotropic or equivalent isotropic displacement parameters $\left(\AA^{2}\right)$

\begin{tabular}{lllll}
\hline & $x$ & $y$ & $z$ & $U_{\text {iso }} / U_{\text {eq }}$ \\
\hline Ni1 & 0.7500 & $0.36655(2)$ & 0.7500 & $0.00475(5)$ \\
Fe1 & 0.5000 & 0.0000 & 0.5000 & $0.00372(6)$ \\
Ca1 & 0.5000 & 0.2500 & $0.08981(7)$ & $0.01187(8)$ \\
P1 & 0.7500 & $0.57298(3)$ & 0.7500 & $0.00385(7)$ \\
P2 & 0.5000 & 0.2500 & $0.58291(8)$ & $0.00327(8)$ \\
O1 & $0.86146(7)$ & $0.49415(6)$ & $0.79418(13)$ & $0.00590(12)$ \\
O4 & $0.61754(11)$ & 0.2500 & $0.73284(17)$ & $0.00587(16)$ \\
O3 & 0.5000 & $0.15625(8)$ & $0.44256(18)$ & $0.00672(17)$ \\
O2 & $0.70724(8)$ & $0.63786(6)$ & $0.93385(12)$ & $0.00762(13)$ \\
\hline
\end{tabular}

Atomic displacement parameters $\left(\AA^{2}\right)$

\begin{tabular}{lllllll}
\hline & $U^{11}$ & $U^{22}$ & $U^{\beta 3}$ & $U^{12}$ & $U^{13}$ & $U^{23}$ \\
\hline Ni1 & $0.00486(9)$ & $0.00326(8)$ & $0.00613(9)$ & 0.000 & $-0.00056(5)$ & 0.000 \\
Fe1 & $0.00264(10)$ & $0.00397(11)$ & $0.00455(11)$ & 0.000 & 0.000 & $-0.00016(8)$ \\
Ca1 & $0.01508(18)$ & $0.01319(18)$ & $0.00735(16)$ & 0.000 & 0.000 & 0.000 \\
P1 & $0.00450(14)$ & $0.00307(14)$ & $0.00398(14)$ & 0.000 & $-0.00041(9)$ & 0.000 \\
P2 & $0.00320(17)$ & $0.00246(17)$ & $0.00414(18)$ & 0.000 & 0.000 & 0.000 \\
O1 & $0.0045(3)$ & $0.0054(3)$ & $0.0079(3)$ & $0.0006(2)$ & $-0.0021(2)$ & $-0.0004(2)$ \\
O4 & $0.0049(4)$ & $0.0057(4)$ & $0.0070(4)$ & 0.000 & $-0.0023(3)$ & 0.000 \\
O3 & $0.0082(4)$ & $0.0045(4)$ & $0.0075(4)$ & 0.000 & 0.000 & $-0.0024(3)$ \\
O2 & $0.0102(3)$ & $0.0069(3)$ & $0.0057(3)$ & $0.0018(2)$ & $0.0001(2)$ & $-0.0020(2)$ \\
\hline
\end{tabular}

Geometric parameters $\left(\AA,{ }^{\circ}\right)$

\begin{tabular}{llll}
\hline $\mathrm{Ni} 1-\mathrm{O} 1$ & $2.0498(8)$ & $\mathrm{Ca} 1-\mathrm{O} 2^{\mathrm{xi}}$ & $2.5987(8)$ \\
$\mathrm{Ni} 1-\mathrm{O} 1^{\mathrm{i}}$ & $2.0499(8)$ & $\mathrm{Ca} 1-\mathrm{O} 2^{\mathrm{xii}}$ & $2.5987(8)$ \\
$\mathrm{Ni} 1-\mathrm{O} 4$ & $2.0529(8)$ & $\mathrm{Ca} 1-\mathrm{O} 2^{\text {xiii }}$ & $2.5987(8)$ \\
$\mathrm{Ni} 1-\mathrm{O} 4^{\mathrm{ii}}$ & $2.0529(8)$ & $\mathrm{Ca} 1-\mathrm{O} 4^{\mathrm{xiv}}$ & $2.5990(12)$ \\
$\mathrm{Ni} 1-\mathrm{O} 2^{\mathrm{iii}}$ & $2.0841(8)$ & $\mathrm{Ca} 1-\mathrm{O} 4^{\mathrm{xv}}$ & $2.5990(12)$ \\
$\mathrm{Ni} 1-\mathrm{O} 2^{\mathrm{iv}}$ & $2.0841(8)$ & $\mathrm{Ca} 1-\mathrm{P} 2$ & $3.1758(7)$ \\
$\mathrm{Fe} 1-\mathrm{O}^{\mathrm{ii}}$ & $1.9504(7)$ & $\mathrm{Ca} 1-\mathrm{P} 2^{\mathrm{xv}}$ & $3.2647(7)$ \\
$\mathrm{Fe} 1-\mathrm{O}^{\mathrm{v}}$ & $1.9504(7)$ & $\mathrm{P} 1-\mathrm{O} 2^{\mathrm{i}}$ & $1.5233(8)$ \\
$\mathrm{Fe} 1-\mathrm{O} 1^{\mathrm{vi}}$ & $1.9504(7)$ & $\mathrm{P} 1-\mathrm{O} 2$ & $1.5233(8)$ \\
$\mathrm{Fe} 1-\mathrm{O} 1^{\mathrm{vii}}$ & $1.9504(7)$ & $\mathrm{P} 1-\mathrm{O} 1^{\mathrm{i}}$ & $1.5719(8)$ \\
$\mathrm{Fe} 1-\mathrm{O} 3^{\text {viii }}$ & $2.0822(11)$ & $\mathrm{P} 1-\mathrm{O} 1$ & $1.5719(8)$ \\
$\mathrm{Fe} 1-\mathrm{O} 3$ & $2.0822(11)$ & $\mathrm{P} 2-\mathrm{O} 3$ & $1.5259(11)$ \\
$\mathrm{Ca} 1-\mathrm{O} 3$ & $2.5832(12)$ & $\mathrm{P} 2-\mathrm{O} 3^{\text {ix }}$ & $1.5259(11)$ \\
$\mathrm{Ca} 1-\mathrm{O} 3^{\mathrm{ix}}$ & $2.5832(12)$ & $\mathrm{P} 2-\mathrm{O} 4^{\text {ix }}$ & $1.5498(11)$
\end{tabular}




\begin{tabular}{|c|c|c|c|}
\hline $\mathrm{Ca} 1-\mathrm{O} 2^{\mathrm{x}}$ & $2.5987(8)$ & $\mathrm{P} 2-\mathrm{O} 4$ & $1.5498(11)$ \\
\hline $\mathrm{O} 1-\mathrm{Ni1}-\mathrm{O} 1^{\mathrm{i}}$ & $70.58(4)$ & $\mathrm{O} 2^{\mathrm{x}}-\mathrm{Ca} 1-\mathrm{O} 2^{\mathrm{xi}}$ & $173.27(4)$ \\
\hline $\mathrm{O} 1-\mathrm{Ni1}-\mathrm{O} 4$ & $171.24(3)$ & $\mathrm{O} 3-\mathrm{Ca} 1-\mathrm{O} 2^{\mathrm{xii}}$ & $77.42(2)$ \\
\hline $\mathrm{O} 1{ }^{\mathrm{i}}-\mathrm{Ni} 1-\mathrm{O} 4$ & $103.13(3)$ & $\mathrm{O} 3^{\mathrm{ix}}-\mathrm{Ca} 1-\mathrm{O} 2^{\mathrm{xii}}$ & $108.72(2)$ \\
\hline $\mathrm{O} 1-\mathrm{Ni} 1-\mathrm{O} 4^{\mathrm{ii}}$ & $103.13(3)$ & $\mathrm{O} 2^{\mathrm{x}}-\mathrm{Ca} 1-\mathrm{O} 2^{\mathrm{xii}}$ & $110.65(3)$ \\
\hline $\mathrm{O} 1^{\mathrm{i}}-\mathrm{Ni} 1-\mathrm{O} 4^{\mathrm{ii}}$ & $171.24(3)$ & $\mathrm{O} 2^{\mathrm{xi}}-\mathrm{Ca} 1-\mathrm{O} 2^{\mathrm{xii}}$ & $68.92(3)$ \\
\hline $\mathrm{O} 4-\mathrm{Ni} 1-\mathrm{O} 4^{\mathrm{ii}}$ & $83.76(5)$ & $\mathrm{O} 3-\mathrm{Ca} 1-\mathrm{O} 2^{\mathrm{xiii}}$ & $108.72(2)$ \\
\hline $\mathrm{O} 1-\mathrm{Ni} 1-\mathrm{O} 2^{\mathrm{iii}}$ & $90.33(3)$ & $\mathrm{O} 3^{\mathrm{ix}}-\mathrm{Ca} 1-\mathrm{O} 2^{\mathrm{xiii}}$ & $77.42(2)$ \\
\hline $\mathrm{O} 1^{\mathrm{i}}-\mathrm{Ni} 1-\mathrm{O} 2^{\mathrm{iii}}$ & $92.27(3)$ & $\mathrm{O} 2^{\mathrm{x}}-\mathrm{Ca} 1-\mathrm{O} 2^{\mathrm{xiii}}$ & $68.92(3)$ \\
\hline $\mathrm{O} 4-\mathrm{Ni} 1-\mathrm{O} 2^{\mathrm{iii}}$ & $83.75(4)$ & $\mathrm{O} 2^{\mathrm{xi}}-\mathrm{Ca} 1-\mathrm{O} 2^{\mathrm{xiii}}$ & $110.65(3)$ \\
\hline 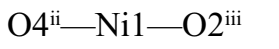 & $93.87(4)$ & $\mathrm{O} 2^{\mathrm{xii}}-\mathrm{Ca} 1-\mathrm{O} 2^{\mathrm{xiii}}$ & $173.27(4)$ \\
\hline $\mathrm{O} 1-\mathrm{Ni} 1-\mathrm{O} 2^{\mathrm{iv}}$ & $92.27(3)$ & $\mathrm{O} 3-\mathrm{Ca} 1-\mathrm{O} 4^{\mathrm{xiv}}$ & $141.08(2)$ \\
\hline $\mathrm{O} 1^{\mathrm{i}}-\mathrm{Ni} 1-\mathrm{O} 2^{\mathrm{iv}}$ & $90.33(3)$ & $\mathrm{O} 3^{\mathrm{ix}}-\mathrm{Ca} 1-\mathrm{O} 4^{\mathrm{xiv}}$ & $141.08(2)$ \\
\hline $\mathrm{O} 4-\mathrm{Ni} 1-\mathrm{O} 2^{\mathrm{iv}}$ & $93.87(4)$ & $\mathrm{O} 2^{\mathrm{x}}-\mathrm{Ca} 1-\mathrm{O} 4^{\mathrm{xiv}}$ & $64.19(3)$ \\
\hline 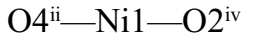 & $83.75(4)$ & $\mathrm{O} 2^{\mathrm{xi}}-\mathrm{Ca} 1-\mathrm{O} 4^{\mathrm{xiv}}$ & $109.37(3)$ \\
\hline  & $176.81(4)$ & $\mathrm{O} 2^{\mathrm{xii}}-\mathrm{Ca} 1-\mathrm{O} 4^{\mathrm{xiv}}$ & $109.37(3)$ \\
\hline $\mathrm{O} 1^{\mathrm{ii}-\mathrm{Fe} 1-\mathrm{O}^{\mathrm{v}}}$ & 180.0 & $\mathrm{O} 2^{\mathrm{xii}}-\mathrm{Ca} 1-\mathrm{O} 4^{\mathrm{xiv}}$ & $64.19(3)$ \\
\hline 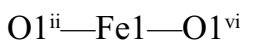 & $85.81(5)$ & $\mathrm{O} 3-\mathrm{Ca} 1-\mathrm{O} 4^{\mathrm{xv}}$ & $141.08(2)$ \\
\hline $\mathrm{O} 1^{\mathrm{v}}-\mathrm{Fe} 1-\mathrm{O} 1^{\mathrm{vi}}$ & $94.19(5)$ & $\mathrm{O} 3^{\mathrm{ix}}-\mathrm{Ca} 1-\mathrm{O} 4^{\mathrm{xv}}$ & $141.08(2)$ \\
\hline $\mathrm{O} 1^{\mathrm{ii}}-\mathrm{Fe} 1-\mathrm{O} 1^{\mathrm{vii}}$ & $94.19(5)$ & $\mathrm{O} 2^{\mathrm{x}}-\mathrm{Ca} 1-\mathrm{O} 4^{\mathrm{xv}}$ & $109.37(3)$ \\
\hline $\mathrm{O} 1^{\mathrm{v}}-\mathrm{Fe} 1-\mathrm{O} 1^{\mathrm{vii}}$ & $85.81(5)$ & $\mathrm{O} 2^{\mathrm{xi}}-\mathrm{Ca} 1-\mathrm{O} 4^{\mathrm{xv}}$ & $64.19(3)$ \\
\hline $\mathrm{O} 1^{\mathrm{vi}}-\mathrm{Fe} 1-\mathrm{O} 1^{\mathrm{vii}}$ & 180.0 & $\mathrm{O} 2^{\mathrm{xii}}-\mathrm{Ca} 1-\mathrm{O} 4^{\mathrm{xv}}$ & $64.19(3)$ \\
\hline $\mathrm{O} 1^{\mathrm{ii}-F e 1-\mathrm{O} 3^{\text {viii }}}$ & $85.29(3)$ & $\mathrm{O} 2^{\mathrm{xiii}}-\mathrm{Ca} 1-\mathrm{O} 4^{\mathrm{xv}}$ & $109.37(3)$ \\
\hline $\mathrm{O} 1^{\mathrm{v}}-\mathrm{Fe} 1-\mathrm{O} 3^{\mathrm{viii}}$ & $94.71(3)$ & $\mathrm{O} 4^{\mathrm{xiv}}-\mathrm{Ca} 1-\mathrm{O} 4^{\mathrm{xv}}$ & $55.60(5)$ \\
\hline $\mathrm{O} 1^{\mathrm{vi}}-\mathrm{Fe} 1-\mathrm{O} 3^{\mathrm{viii}}$ & $94.71(3)$ & $\mathrm{O} 2{ }^{\mathrm{i}}-\mathrm{P} 1-\mathrm{O} 2$ & $112.08(6)$ \\
\hline $\mathrm{O} 1^{\mathrm{vii}}-\mathrm{Fe} 1-\mathrm{O} 3^{\text {viii }}$ & $85.29(3)$ & $\mathrm{O} 2^{\mathrm{i}}-\mathrm{P} 1-\mathrm{O} 1^{\mathrm{i}}$ & $116.00(4)$ \\
\hline $\mathrm{O} 1 \mathrm{ii}-\mathrm{Fe} 1-\mathrm{O} 3$ & $94.71(3)$ & $\mathrm{O} 2-\mathrm{P} 1-\mathrm{O} 1^{\mathrm{i}}$ & $107.24(4)$ \\
\hline $\mathrm{O} 1^{\mathrm{v}}-\mathrm{Fe} 1-\mathrm{O} 3$ & $85.29(3)$ & $\mathrm{O} 2{ }^{\mathrm{i}}-\mathrm{P} 1-\mathrm{O} 1$ & $107.24(4)$ \\
\hline $\mathrm{O} 11^{\mathrm{vi}-\mathrm{Fe} 1-\mathrm{O} 3}$ & $85.29(3)$ & $\mathrm{O} 2-\mathrm{P} 1-\mathrm{O} 1$ & $116.00(4)$ \\
\hline $\mathrm{O} 1{ }^{\mathrm{vii}}-\mathrm{Fe} 1-\mathrm{O} 3$ & $94.71(3)$ & $\mathrm{O} 1{ }^{\mathrm{i}}-\mathrm{P} 1-\mathrm{O} 1$ & $97.76(6)$ \\
\hline $\mathrm{O} 3^{\text {viii- }-\mathrm{Fe} 1-\mathrm{O} 3}$ & $180.000(10)$ & $\mathrm{O} 3-\mathrm{P} 2-\mathrm{O} 3^{\mathrm{ix}}$ & $107.35(9)$ \\
\hline $\mathrm{O} 3-\mathrm{Ca} 1-\mathrm{O} 3^{\mathrm{ix}}$ & $56.84(5)$ & $\mathrm{O} 3-\mathrm{P} 2-\mathrm{O} 4^{\mathrm{ix}}$ & $111.66(3)$ \\
\hline $\mathrm{O} 3-\mathrm{Ca} 1-\mathrm{O} 2^{\mathrm{x}}$ & $77.42(2)$ & $\mathrm{O} 3^{\mathrm{ix}}-\mathrm{P} 2-\mathrm{O} 4^{\mathrm{ix}}$ & $111.66(3)$ \\
\hline $\mathrm{O} 3^{\mathrm{ix}}-\mathrm{Ca} 1-\mathrm{O} 2^{\mathrm{x}}$ & $108.72(2)$ & $\mathrm{O} 3-\mathrm{P} 2-\mathrm{O} 4$ & $111.66(3)$ \\
\hline $\mathrm{O} 3-\mathrm{Ca} 1-\mathrm{O} 2^{\mathrm{xi}}$ & $108.72(2)$ & $\mathrm{O} 3^{\mathrm{ix}-\mathrm{P} 2-\mathrm{O} 4}$ & $111.66(3)$ \\
\hline $\mathrm{O} 3^{\mathrm{ix}}-\mathrm{Ca} 1-\mathrm{O} 2^{\mathrm{xi}}$ & $77.42(2)$ & $\mathrm{O} 4^{\mathrm{ix}}-\mathrm{P} 2-\mathrm{O} 4$ & $102.91(9)$ \\
\hline
\end{tabular}

Symmetry codes: (i) $-x+3 / 2, y,-z+3 / 2$; (ii) $-x+3 / 2,-y+1 / 2,-z+3 / 2$; (iii) $x,-y+1,-z+2$; (iv) $-x+3 / 2,-y+1, z-1 / 2$; (v) $x-1 / 2, y-1 / 2, z-1 / 2$; (vi) $-x+3 / 2$, $y-1 / 2, z-1 / 2$; (vii) $x-1 / 2,-y+1 / 2,-z+3 / 2$; (viii) $-x+1,-y,-z+1$; (ix) $-x+1,-y+1 / 2, z$; (x) $-x+1, y-1 / 2,-z+1$; (xi) $x,-y+1,-z+1$; (xii) $x, y-1 / 2,-z+1$; (xiii) $-x+1,-y+1,-z+1$; (xiv) $-x+1,-y+1 / 2, z-1$; (xv) $x, y, z-1$. 